\title{
Large Time Behavior of Entropy Solutions to Two-Dimensional Unipolar Hydrodynamic Model for Semiconductor Devices with Variable Coefficient Damping
}

\author{
Lili Chen \\ Department of Mathematics, Shandong Normal University, Jinan 250014, Shandong, China.
}

How to cite this paper: Lili Chen. (2022) Large Time Behavior of Entropy Solutions to Two-Dimensional Unipolar Hydrodynamic Model for Semiconductor Devices with Variable Coefficient Damping. Journal of Applied Mathematics and Computation, 6(1), 24-29.

DOI: 10.26855/jamc.2022.03.005

Received: January 6, 2022

Accepted: January 30, 2022

Published: February 22, 2022

*Corresponding author: Lili Chen, Department of Mathematics, Shandong

Normal University, Jinan 250014,

Shandong, China.

Email: clili0099@163.com

\begin{abstract}
This paper mainly studies the large time behavior of two-dimensional isothermal spherically symmetric compressible Euler-Poisson equations with variable coefficient damping in a bounded region. This equation and its variants have been used to describe the dynamic behavior of many important physical flows including the propagation of electrons in submicron semiconductor devices, the biological transport of ions for channel proteins, the motion of stars in the theory of general relativity and so on. This paper mainly proves that the weak solution converges exponentially to the unique stationary solution in time. The main methods are entropy estimation and energy method. Here, the key step is to construct an appropriate entropy estimation to cooperate with the energy method to obtain that when $t \rightarrow \infty$, the spherically symmetric weak solution of the isothermal spherically symmetric Euler-Poisson equations with variable coefficient damping converges to the unique smooth solution of the corresponding stationary equations at an exponential rate under the condition that the corresponding initial values are satisfied.
\end{abstract}

\section{Keywords}

Euler-Poisson equations, Damping, Large time behavior, Weak entropy solution

\section{Introduction}

In this paper, we consider the large time behavior of two-dimensional isothermal spherically symmetric compressible Euler-Poisson equations with variable coefficient damping.

The Euler-Poisson equations for compressible isothermal fluids are of the form:

$$
\left\{\begin{array}{c}
\rho_{t}+\nabla \cdot \vec{m}=0 \\
\vec{m}_{t}+\nabla\left(\frac{\vec{m} \otimes \vec{m}}{\rho}\right)+\nabla p(\rho)=\rho \nabla \phi+H(\vec{x}, t) \vec{m}, \\
\Delta \phi=\rho-D(\vec{x}),
\end{array}\right.
$$

where space variable $\vec{x} \in R^{N}$, and $L_{1} \leq|\vec{x}| \leq L_{2}$ ( $L_{1}$ and $L_{2}$ are two positive constants), time variable $t \in[0,+\infty$ ). Here, $\rho \geq 0, m, p(\rho)$ and $\nabla \phi$ denote the electron density, electron current density, pressure, and electric filed, respectively. The pressure $p(\rho)=\rho$ for the isothermal fluid. $D(\vec{x}) \geq 0$ is the doping profile. $H(\vec{x}, t)$ represents the damping coefficient.

We are interested in the radially symmetric solutions to system (1.1) with the form 


$$
(\rho, \vec{m}, \phi)(\vec{x}, t)=\left(\rho(x, t), m(x, t) \frac{\vec{x}}{x}, \phi(x, t)\right), x=|\vec{x}| .
$$

Then $(\rho(x, t), m(x, t), \phi(x, t))$ in (1.2) is governed by the one-dimensional Euler-Poisson equations with geometric source terms:

$$
\left\{\begin{array}{c}
\rho_{\mathrm{t}}+m_{x}=-\frac{N-1}{x} m \\
m_{t}+\left(\frac{m^{2}}{\rho}+\rho\right)_{x}=-\frac{N-1}{x} \frac{m^{2}}{\rho}+H(x, t) m+\rho \phi_{x}, \\
\phi_{x x}=-\frac{N-1}{x} \phi_{x}+\rho-D(x), x \in\left[L_{1}, L_{2}\right], t \in[0,+\infty),
\end{array}\right.
$$

where the doping profile $D(\vec{x})=D(x)$, and damping coefficient $H(\vec{x}, t)=H(x, t)$ are radial symmetric functions.

We assume that $D(x)$ satisfies

$$
D(x) \epsilon C\left[L_{1}, L_{2}\right], D^{*}=\underset{x}{\sup } D(x) \geq \underset{x}{\inf } D(x)=D_{*}>0,
$$

Where $D^{*}$ and $D_{*}$ are two positive constants.

First, to make full use of the law of conservation of mass, we use following transformation:

$$
n=x^{N-1} \rho, J=x^{N-1} m, E=x^{N-1} \phi_{x} .
$$

Then, $n, J, E$ satisfies the equations

$$
\left\{\begin{array}{c}
n_{t}+J_{x}=0 \\
J_{t}+\left(\frac{J^{2}}{n}+n\right)_{x}=\frac{N-1}{x} n+H(x, t) J+\frac{n E}{x^{N-1}} \\
E_{x}=n-x^{N-1} D(x):=n-b(x) .
\end{array}\right.
$$

We consider the initial boundary value condition

$$
\begin{aligned}
& \left.(n, J)\right|_{t=0}=\left(n_{0}(x), J_{0}(x)\right), L_{1} \leq x \leq L_{2}, \\
& J\left(L_{1}, t\right)=J\left(L_{2}, t\right)=0, E\left(L_{1}\right)=E\left(L_{2}\right)=-1 .
\end{aligned}
$$

Obviously, the first two equations of system (1.6) are typical 1-D system of balance laws. We can rewrite them into the following form:

$$
\left\{\begin{aligned}
v_{t}+F(v)_{x}= & G(x, v), x \in\left[L_{1}, L_{2}\right], t \in[0,+\infty) \\
& \left.v\right|_{t=0}=v_{0}(x)
\end{aligned}\right.
$$

where $v=(n, J)^{T}, \nabla F=\left(\begin{array}{cc}0 & 1 \\ -\frac{J^{2}}{n^{2}}+1 & \frac{2 J}{n}\end{array}\right)$ and the source term $G(x, v)=\left(0, \frac{N-1}{x} n+H(x, t) J+\frac{n E}{x^{N-1}}\right)^{T}$.

In recent years, a lot of research has been carried out on Euler-Poisson systems in bounded and unbounded regions. For large time behavior, Huang-Pan-Yu [1] first studied the large time behavior of weak entropy solutions of Euler-Poisson systems, and established a large time behavior framework of arbitrary uniformly bounded weak entropy solutions, assuming that the upper bound of density is independent of time $t$. Yu [2] obtained the large time behavior of two-dimensional spherically symmetric Euler-Poisson system with constant coefficient damping. Then, when $\gamma>3$, Yu [3] improved the result in [1] and extended it to that the density can increase slowly with time, i.e. $\|\rho(x, t)\|_{L^{\infty}} \leq C t^{2}$. Other results are shown in [4]-[12].

In [2], Yu studied the large time behavior of spherically symmetric weak entropy solutions of Euler-Poisson system with dimension $N=2$ and constant damping coefficient. Here, we generalize the results and prove the large time behavior of spherically symmetric weak entropy solutions of Euler-Poisson system with variable damping coefficients when dimension $N=2$.

Before introducing the main theorems in this paper, we first give the definition of weak entropy solution and stationary solution when $N=2$.

Definition 1.1 A bounded measurable vector function $v(x, t)$ is called a global entropy solution of system (1.8) in $\Pi=\left\{(x, t) \mid L_{1} \leq x \leq L_{2}, t \geq 0\right\}$, if $v(x, t)$ satisfies the following:

(i) System (1.8) satisfies in the sense of distributions;

(ii) The entropy inequality holds in the sense of distributions, i.e.,

$$
\eta_{t}+q_{x}-\nabla \eta \cdot G(x, v) \leq 0 \quad \text { in } D^{\prime}
$$

for any weak entropy-entropy flux pair $(\eta, q)$ with convex $\eta(v)$, satisfying 


$$
\nabla q(v)=\nabla \eta(v) \nabla F .
$$

Definition 1.2 The stationary solution of problems (1.6) (1.7) is the smooth solution of

$$
\left\{\begin{array}{c}
\tilde{J}_{x}=0, \\
\left(\frac{\tilde{J}^{2}}{\tilde{n}}+\tilde{n}\right)_{x}=\frac{\tilde{n}}{x}+H(x, t) \tilde{J}+\frac{\tilde{n} \tilde{E}}{x} \\
\tilde{E}_{x}=\tilde{n}-b(x),
\end{array}\right.
$$

with the boundary condition

$$
\begin{gathered}
\tilde{J}\left(L_{1}\right)=\tilde{J}\left(L_{2}\right)=0, L_{1} \leq x \leq L_{2}, \\
\tilde{E}\left(L_{1}\right)=\tilde{E}\left(L_{2}\right)=-1 .
\end{gathered}
$$

Using the viscosity vanishing method and compensation compactness, we can prove the existence of global entropy weak solutions. This result will be proved in another article, and we omit it here. Throughout this paper, we assume:

(H1) Let $(n, J, E)(x, t)$ be any globally defined entropy weak solution of (1.6) (1.7) and the density $n(x, t)$ satisfies

$$
0 \leq n(x, t) \leq C_{0},(x, t) \in \Pi
$$

for certain positive constant $C_{0}$.

Since $\tilde{J}=0$, according to the results in [2], for the stationary problem (1.10), we have the following existence and uniqueness theorem:

Theorem 1.1 We assume $b(x) \in L^{2}\left[L_{1}, L_{2}\right]$ and satisfy

$$
0<b_{*} \leq b(x) \leq b^{*}, x \in\left[L_{1}, L_{2}\right] .
$$

Then the stationary equations (1.10) has a unique solution $(\tilde{n}(x), \tilde{E}(x))$ on $C^{1}\left[L_{1}, L_{2}\right] \times C^{1}\left[L_{1}, L_{2}\right]$ and satisfies

$$
0<b_{*} \leq \tilde{n}(x) \leq b^{*}, x \in\left[L_{1}, L_{2}\right],
$$

where $b *$ and $b *$ are two positive constants.

Now, we introduce new variables.

$$
y(x, t)=-\int_{L_{1}}^{x}(n(s, t)-\tilde{n}(s)) d s=-(E-\tilde{E})(x, t), x \in\left[L_{1}, L_{2}\right] .
$$

Then

$$
y_{x}=-(n-\tilde{n})(x, t), y_{t}=J(x, t)
$$

The relative entropy flux pair is defined as

$$
\begin{gathered}
\eta_{*}(n, J)=\frac{J^{2}}{2 n}+n \ln n-\tilde{n} \ln \tilde{n}-(\ln \tilde{n}+1)(n-\tilde{n}), \\
q_{*}(n, J)=\frac{J^{3}}{2 n^{2}}+(\ln n+1) J-(\ln \tilde{n}+1) J .
\end{gathered}
$$

Then we give our main result in Section 3 as follows.

Theorem 1.2 (Large time behavior) Suppose $N=2$, denote $(n, J, E)(x, t)$ is the global entropy solution of (1.6) (1.7), $(\tilde{n}(x), \tilde{E}(x))$ is the stationary solution, $b(x) \in L^{2}\left[L_{1}, L_{2}\right]$, and

$$
0<b_{*} \leq b(x) \leq b^{*}, x \in\left[L_{1}, L_{2}\right]
$$

Assume the initial value $\left(n_{0}(x), J_{0}(x)\right)$ satisfied

$$
\begin{gathered}
\int_{L_{1}}^{L_{2}}\left(n_{0}(x)-b(x)\right) d x=0, \\
y(x, 0) \epsilon L^{2}\left[L_{1}, L_{2}\right], \int_{L_{1}}^{L_{2}} \eta_{*}(x, 0) d x<\infty .
\end{gathered}
$$

The damping coefficient meet $H(x, t)<-\delta, H_{t}(x, t)>\frac{1}{L_{1}^{2}}+\frac{2}{L_{1}}\left(\frac{L_{2}-1}{2 L_{2}} b^{*}-\frac{7 L_{1}+1}{6 L_{1}} b_{*}\right)$, where $\delta$ is a small positive constant. Then, it holds that

$$
\int_{L_{1}}^{L_{2}}\left(y^{2}+y_{t}{ }^{2}+y_{x}^{2}\right) d x \leq C e^{-\tilde{C t}} \int_{L_{1}}^{L_{2}}\left(\eta_{*}(x, 0)+y^{2}(x, 0)\right) d x,
$$

for some positive constants Cand $\tilde{C}$. 
Remark 1.1 $H(x, t)<-\delta$ indicates that it is a damping. $H_{t}(x, t)>\frac{1}{L_{1}^{2}}+\frac{2}{L_{1}}\left(\frac{L_{2}-1}{2 L_{2}} b^{*}-\frac{7 L_{1}+1}{6 L_{1}} b_{*}\right)$ indicates that the damping cannot be too weak. For example, when $t \rightarrow \infty, H(x, t) \rightarrow 0$, this condition is not feasible.

\section{Preliminaries}

We first consider the following homogeneous system of conservation laws:

The eigenvalues are

$$
\left\{\begin{array}{c}
n_{t}+J_{x}=0 \\
J_{t}+\left(\frac{J^{2}}{n}+n\right)_{x}=0
\end{array}\right.
$$

and the corresponding right eigenvectors are

$$
\lambda_{1}=\frac{J}{n}-1, \lambda_{2}=\frac{J}{n}+1
$$

The Riemann invariants $(w, z)$ are given by

$$
r_{1}=\left[\begin{array}{c}
1 \\
\lambda_{1}
\end{array}\right], r_{2}=\left[\begin{array}{c}
1 \\
\lambda_{2}
\end{array}\right]
$$

$$
\mathrm{w}=\frac{J}{n}+\ln n, z=\frac{J}{n}-\ln n,
$$

satisfying $\nabla w \cdot r_{1}=0$ and $\nabla z \cdot r_{2}=0$, where $\nabla=\left(\partial_{n}, \partial_{J}\right)$ is the gradient with respect to $(n, J)$.

The mechanical energy $\eta^{*}(n, J)$ and mechanical energy flux $q^{*}(n, J)$ have the following formula

$$
\eta^{*}(n, J)=\frac{J^{2}}{2 n}+n \ln n, q^{*}(n, J)=\frac{J^{3}}{2 n^{2}}+J \ln n .
$$

\section{Large time behavior}

In this section, our aim is to prove theorem 1.2. We need to prove that when $t \rightarrow \infty$, Under condition (1.18), the spherically symmetric weak entropy solution $(n, J, E)$ of $(1.6)(1.7)$ converges to the unique smooth solution $(\tilde{n}, \tilde{J}, \tilde{E})$ of (1.10) (1.11) at an exponential rate. Combining (1.6) and (1.10), we know that $y$ satisfies the following equation:

$$
y_{t t}+\left(\frac{J^{2}}{n}\right)_{x}-y_{x x}-H(x, t) y_{t}-\frac{y y_{x}-\tilde{E} y_{x}-\tilde{n} y}{x}+\frac{y_{x}}{x}=0 \text {. }
$$

The corresponding boundary conditions are

$$
\begin{gathered}
y\left(L_{2}, t\right)=-\int_{L_{1}}^{L_{2}}(n(x, t)-\tilde{n}(x)) d x=-\int_{L_{1}}^{L_{2}}\left(n_{0}(x)-\tilde{n}(x)\right) d x=-\int_{L_{1}}^{L_{2}}\left(n_{0}(x)-b(x)+b(x)-\tilde{n}(x)\right) d x \\
=-\int_{L_{1}}^{L_{2}}\left(n_{0}(x)-b(x)\right) d x+\tilde{E}\left(L_{2}\right)-\tilde{E}\left(L_{1}\right)=0=y\left(L_{1}, t\right) .
\end{gathered}
$$

Multiplyyby (3.1) and then integrate on $\left[L_{1}, L_{2}\right]$ to get

i.e.,

$$
\begin{aligned}
& \frac{\mathrm{d}}{\mathrm{d} t} \int_{L_{1}}^{L_{2}}\left(y y_{t}-\frac{H(x, t)}{2} y^{2}\right) d x+\int_{L_{1}}^{L_{2}} y_{x}^{2} d x-\int_{L_{1}}^{L_{2}} y_{t}^{2} d x-\int_{L_{1}}^{L_{2}} \frac{J^{2}}{n} y_{x} d x+\int_{L_{1}}^{L_{2}} \frac{H_{t}(x, t)}{2} y^{2} d x \\
& =\int_{L_{1}}^{L_{2}} \frac{y^{2} y_{x}-\tilde{E} y y_{x}-\tilde{n} y^{2}}{x} d x-\int_{L_{1}}^{L_{2}} \frac{y y_{x}}{x} d x,
\end{aligned}
$$

where

$$
\begin{aligned}
& \frac{\mathrm{d}}{\mathrm{d} t} \int_{L_{1}}^{L_{2}}\left(y y_{t}-\frac{H(x, t)}{2} y^{2}\right) d x+\int_{L_{1}}^{L_{2}} y_{x}^{2} d x \\
& =\int_{L_{1}}^{L_{2}} y_{t}^{2} d x+\int_{L_{1}}^{L_{2}} \frac{J^{2}}{n} y_{x} d x-\int_{L_{1}}^{L_{2}} \frac{H_{t}(x, t)}{2} y^{2} d x-\int_{L_{1}}^{L_{2}} \frac{y^{2}}{2 x^{2}} d x \\
& +\int_{L_{1}}^{L_{2}} \frac{y^{2}}{x^{2}}\left(\frac{y}{3 x}-\tilde{n}+\frac{\tilde{E}_{x}}{2}-\frac{\tilde{E}}{2 x}\right) d x
\end{aligned}
$$




$$
\begin{aligned}
\frac{y}{3 x}-\tilde{n}+\frac{\tilde{E}_{x}}{2}-\frac{\tilde{E}}{2 x} & =-\frac{\tilde{n}+b(x)}{2}-\frac{1}{3 x} \int_{L_{1}}^{x}(n-\tilde{n}) d x-\frac{1}{2 x}\left(\int_{L_{1}}^{x}(\tilde{n}-b(x)) d x-1\right) \\
& \leq \frac{1}{3 x} \int_{L_{1}}^{x}(b(x)-n) d x-\frac{1}{6 x} \int_{L_{1}}^{x}(\tilde{n}-b(x)) d x-b_{*}+\frac{1}{2 x} \\
& \leq-b_{*}+\frac{1}{3 x}(x-1) b^{*}+\frac{1}{6 x}(x-1)\left(b^{*}-b_{*}\right)+\frac{1}{2 x} \\
& \leq \frac{L_{2}-1}{2 L_{2}} b^{*}-\frac{7 L_{1}+1}{6 L_{1}} b_{*}+\frac{1}{2 L_{1}}
\end{aligned}
$$

so,

$$
\begin{aligned}
& -\frac{H_{t}(x, t)}{2}-\frac{1}{2 x^{2}}+\frac{1}{x}\left(\frac{y}{3 x}-\tilde{n}+\frac{\tilde{E}_{x}}{2}-\frac{\tilde{E}}{2 x}\right) \\
& \leq-\frac{H_{t}(x, t)}{2}+\frac{1}{2 L_{1}^{2}}+\frac{1}{x}\left(\frac{L_{2}-1}{2 L_{2}} b^{*}-\frac{7 L_{1}+1}{6 L_{1}} b_{*}\right) \\
& \leq-\frac{H_{t}(x, t)}{2}+\frac{1}{2 L_{1}^{2}}+\frac{1}{L_{1}}\left(\frac{L_{2}-1}{2 L_{2}} b^{*}-\frac{7 L_{1}+1}{6 L_{1}} b_{*}\right) .
\end{aligned}
$$

And because we already know

$$
H_{t}(x, t)>\frac{1}{L_{1}^{2}}+\frac{2}{L_{1}}\left(\frac{L_{2}-1}{2 L_{2}} b^{*}-\frac{7 L_{1}+1}{6 L_{1}} b_{*}\right) .
$$

Then there exists $\sigma>0$ such that

$$
-\frac{H_{t}(x, t)}{2}-\frac{1}{2 x^{2}}+\frac{1}{x}\left(\frac{y}{3 x}-\tilde{n}+\frac{\tilde{E}_{x}}{2}-\frac{\tilde{E}}{2 x}\right) \leq-\sigma .
$$

Thus, we can rewrite (3.4) as follows:

$$
\frac{\mathrm{d}}{\mathrm{d} t} \int_{L_{1}}^{L_{2}}\left(y y_{t}-\frac{H(x, t)}{2} y^{2}\right) d x+\int_{L_{1}}^{L_{2}} y_{x}^{2} d x+\sigma \int_{L_{1}}^{L_{2}} y^{2} d x \leq \int_{L_{1}}^{L_{2}} y_{t}{ }^{2} d x+\int_{L_{1}}^{L_{2}} \frac{J^{2}}{n} y_{x} d x=\int_{L_{1}}^{L_{2}} \frac{\widetilde{\mathrm{n}}}{n} y_{t}{ }^{2} d x, \forall x \in\left[L_{1}, L_{2}\right] .
$$

In addition, according to definitions (1.16) and (1.17) of $\eta^{*}$ and $q^{*}$, we have the following inequality holds in the sense of distribution:

$$
\eta_{* t}+q_{* x}+\frac{\tilde{n}_{x}}{\tilde{n}} J-\frac{J E}{x}-\frac{H(x, t) J^{2}}{n}-\frac{J}{x} \leq 0 .
$$

We notice that

$$
\frac{J E}{x}=\frac{J \tilde{n}_{x}}{\tilde{n}}-\frac{J}{x}-\frac{y y_{t}}{x}
$$

Putting (3.11) into (3.10), we have

$$
\eta_{* t}+q_{* x}-\frac{H(x, t) J^{2}}{n}+\frac{y y_{t}}{x} \leq 0 .
$$

Integrate (3.12) on interval $\left[L_{1}, L_{2}\right]$ to arrive at

$$
\frac{d}{d t} \int_{L_{1}}^{L_{2}}\left(\eta_{*}+\frac{y^{2}}{2 x}\right) d x-\int_{L_{1}}^{L_{2}} \frac{H(x, t) J^{2}}{n} d x \leq 0 .
$$

Let $\Lambda$ sufficiently big so that $\Lambda>\frac{b^{*}}{\delta_{0}}+\|n\|_{L^{\infty}}+1$, multiply (3.13) by $\Lambda$ and add the result to (3.9), we have

$$
\frac{d}{d t} \int_{L_{1}}^{L_{2}}\left(\Lambda \eta_{*}+\left(\frac{\Lambda}{2 x}-\frac{H(x, t)}{2}\right) y^{2}+y y_{t}\right) d x+\int_{L_{1}}^{L_{2}}\left(y_{x}^{2}+\sigma y^{2}+\frac{-\Lambda H-\widetilde{n}}{n} y_{t}^{2}\right) d x \leq 0
$$

Since

$$
-\Lambda H-\tilde{n}>\left(\frac{b^{*}}{\delta_{0}}+\|n\| L_{\infty}+1\right) \delta_{0}-b^{*}>\|n\| L_{\infty} \delta_{0}
$$

so there exists $\tilde{C}$ such that

$$
\frac{d}{d t} \int_{L_{1}}^{L_{2}}\left(\Lambda \eta_{*}+\left(\frac{\Lambda}{2 x}-\frac{H(x, t)}{2}\right) y^{2}+y y_{t}\right) d x+\tilde{C} \int_{L_{1}}^{L_{2}}\left(y_{x}{ }^{2}+y^{2}+\frac{y_{t}{ }^{2}}{n}\right) d x \leq 0 .
$$

We define 


$$
\eta_{*}=Q_{1}+Q_{2}, Q_{1}=\frac{J^{2}}{2 n}, Q_{2}=n \ln n-\tilde{n} \ln \tilde{n}-(\ln \tilde{n}+1)(n-\tilde{n}) .
$$

Obviously, $Q_{2}$ is the quadratic remainder of $n \ln n$ according to the Taylor expansion of $\tilde{n}$, because of the convexity of $n \ln n$, we know that $Q_{2}$ is nonnegative, so there are two positive constants $C_{1}$ and $C_{1}$ such that

$$
C_{1}\left(y_{x}^{2}+y_{t}^{2}\right) \leq \eta_{*} \leq C_{2}\left(y_{x}^{2}+\frac{y_{t}^{2}}{n}\right)
$$

And $x \in\left[L_{1}, L_{2}\right]$, then there exists two positive constants $C_{3}$ and $C_{4}$ such that

We set

$$
C_{3}\left(y^{2}+y_{x}^{2}+y_{t}^{2}\right) \leq \Lambda \eta_{*}+\left(\frac{\Lambda}{2 x}-\frac{H(x, t)}{2}\right) y^{2}+y y_{t} \leq C_{4}\left(y^{2}+\eta_{*}\right)
$$

$$
F(t)=\int_{L_{1}}^{L_{2}}\left(\Lambda \eta_{*}+\left(\frac{\Lambda}{2 x}-\frac{H(x, t)}{2}\right) y^{2}+y y_{t}\right) d x
$$

Then we can rewrite (3.16) into the following form:

$$
\frac{d}{d t} F(t)+C_{5} F(t) \leq 0
$$

Using Gronwall inequality for (3.17), we obtain

$$
C_{3} \int_{L_{1}}^{L_{2}}\left(y^{2}+y_{x}^{2}+y_{t}^{2}\right)(x, t) d x \leq F(t) \leq F(0) e^{-C_{5} t} \leq C_{4} e^{-C_{5} t} \int_{L_{1}}^{L_{2}}\left(y^{2}+\eta_{*}\right)(x, 0) d x .
$$

This completes the proof of the Theorem 1.2.

\section{References}

[1] F. M. Huang, R. H. Pan, H. M. Yu. (2008). Large time behavior of Euler-Poisson system for semiconductor. Sci. China Ser. A., 51, 965-972.

[2] H. M. Yu. (2010). Large time behavior of Euler-Poisson equations for isothermal fluids with spherical symmetry. J. Math. Anal. Appl., 363, 302-309.

[3] H. M. Yu. (2015). Large time behavior of entropy solutions to a unipolar hydrodynamic model of Semiconductors. Comm. Math. Sci., 14, 69-82.

[4] L. Hsiao, T. Yang. (2001). Asymptotics of initial boundary value problems for hydrodynamic and driftdiffusion models for semiconductors. J. Differ. Equ., 170, 472-493.

[5] F. M. Huang, M. Mei, Y. Wang, H. M. Yu. (2011). Asymptotic convergence to planar stationary waves for multi-dimensional unipolar hydrodynamic model of semiconductors. J. Differ. E-qu., 251, 1305-1331.

[6] G. Q. Chen, T. H. Li. (2003). Global entropy solutions in linfinity to the Euler equations and Euler-Poisson equations for isothermal fluids with spherical symmetry. Methods Appl. Anal., 10, 215-244.

[7] Y. Guo. (1998). Smooth irrotational flows in the large to the Euler-Poisson system in R3+1. Commun. Math. Phys., 195, 249-265.

[8] F. M. Huang, Z. Wang. (2003). Convergence of viscosity solutions for isentropic gas dynamics. SIAMJ. Math. Anal., 34, 595-610.

[9] F. M. Huang, T. H. Li, H. M. Yu, D. F. Yuan. (2018). Large time behavior of entropy solutions to one-dimensional unipolar hydrodynamic model for semiconductor devices. Z. Angew. Math. Phys., 69, 69.

[10] Y. G. Lu. (2011). Resonance for the isothermal system of isentropic gas dynamics. Proc. Amer. Math. Soc., 139, 2821-2826.

[11] Y. G. Lu. (2002). Hyperbolic conservation laws and the compensated compactness method. CRC Press.

[12] H. M. Yu, Y. L. Zhan. (2016). Large time behavior of solutions to multi-dimensional bipolar hydrodynamic model of semiconductors with vacuum. J. Math. Anal. Appl., 438, 856-874. 